\title{
Density profile and turbulence evolution during L-H transition studied with the ultra-fast swept reflectometer on ASDEX Upgrade
}

\author{
A. Medvedeva ${ }^{1,2,3,4}$, C. Bottereau ${ }^{2}$, F. Clairet $^{2}$, P. Hennequin ${ }^{5}$, \\ U. Stroth ${ }^{1,4}$, G. Birkenmeier ${ }^{1,4}$, M. Cavedon ${ }^{1}$, G. D. Conway ${ }^{1}$, \\ T. Happel ${ }^{1}$, S. Heuraux ${ }^{3}$, D. Molina ${ }^{2}$, A. Silva ${ }^{6}$, \\ M. Willensdorfer ${ }^{1}$, ASDEX Upgrade Team $^{1}$ \\ ${ }^{1}$ Max-Planck-Institut für Plasmaphysik, 85748 Garching, Germany \\ ${ }^{2}$ CEA, IRFM, 13108 St-Paul-Lez-Durance, France \\ ${ }^{3}$ Institut Jean Lamour UMR 7198 CNRS, Université de Lorraine, 54000 Nancy, \\ France \\ ${ }^{4}$ Physik-Department E28, Technische Universität München, 85747 Garching, \\ Germany \\ ${ }^{5}$ LPP, CNRS, Ecole polytechnique, UPMC Univ. Paris 06, Univ. Paris-Sud, \\ Observatoire de Paris, Universit Paris-Saclay, Sorbonne Universits, PSL Research \\ University, 91128 Palaiseau, France \\ ${ }^{6}$ Instituto de Plasmas e Fusão Nuclear, IST, Universidade Lisboa, Lisbon, Portugal
}

\begin{abstract}
The ultra-fast swept reflectometer with the sweep time of $1 \mu \mathrm{s}$, inferior to the characteristic turbulent time scale, has provided the measurements of the fast density and density fluctuation evolution across major parts of tokamak plasma radius. The L-H transitions in a series of plasma discharges in ASDEX Upgrade have been studied with a high temporal resolution. The comparison of the density fluctuation behaviour in L- and $\mathrm{H}$-mode is presented. The I-phase oscillation dynamics has been described in terms of the density fluctuation level, the radial electric field and the normalised electron pressure gradient. Indications for a phase shift between the turbulence and the radial electric field are observed in the beginning of the I-phase, where the turbulence grows first and the radial electric field increase follows. In the established I-phase the electric field and the turbulence are in phase.
\end{abstract}

Keywords: magnetic confinement, plasma physics, fusion, turbulence, L-H transition, I-phase 


\section{Introduction}

The plasma confinement is limited by the radial energy and particle transport, in which turbulence plays an important role. Hence for fusion plasmas regions of reduced turbulence, such as transport barriers, are of special importance. The most pronounced transport barrier develops at the plasma edge at the transition from the low (L-) to the high (H-) confinement mode [1]. According to one of the most widely accepted theories concerning the transition from the $\mathrm{L}$ to $\mathrm{H}$ mode, the plasma edge turbulence is stabilized by increased radial electric field gradient: the $\mathbf{E}_{\mathbf{r}} \times \mathbf{B}$ flow shear reduces turbulence and decreases the radial size of turbulent structures [2]. A transport barrier forms in the edge where the density, the temperature together with their gradients increase.

Between the L- and the H-mode an intermediate phase, called I-phase [3], occurs, where the turbulence level, background and turbulent flows oscillate in the kilohertz range and the radial electric field evolves to the H-mode values. The I-phase has been observed in different machines [3, 4, 5, 6, 7, 8, 9]. Experimentally the I-phase can be characterised by the oscillations of the $\mathbf{E}_{\mathbf{r}} \times \mathbf{B}$ flow and the turbulence level, moreover similar oscillations have been observed in the plasma density, temperature, their gradients and magnetic fluctuations from Mirnov coils. In ASDEX Upgrade (AUG) the number of periods preceding an established H-mode varies with the heating power and density, but at least a few oscillations are always present in favorable magnetic configuration (ion $\nabla B$ drift in X-point direction) [10]. The origin of the strong radial electric field appearing in the I-phase and in the H-mode is still discussed. In principle it can have contributions from the background electric field, as described by neoclassical theory, as well as from the turbulence driven zonal flows.

Neglecting various forces which might have influence on the $E_{r}$ (such as ion orbit loss [11]), it is often supposed that the radial electric field can be written as a sum of two contributions:

$$
E_{r}=E_{r}^{n e o}+E_{r}^{Z F}=v_{E \times B} B
$$

where

$$
E_{r}^{\text {neo }}=\frac{\nabla p_{i}}{Z e n_{i}}-\alpha \frac{\nabla T_{i}}{e}+\frac{B}{B_{\phi}} B_{\theta}\left\langle V_{i \|}\right\rangle
$$

is the neoclassical or the mean background electric field, $E_{r}^{Z F}$ is the radial electric field created by the zonal flow through the Reynolds stress, $T_{i}, n_{i}$ and $p_{i}$ are the ion temperature, density and pressure, $B$ is the magnetic field strength and $\alpha$ is a coefficient depending on collisionality [12]. The neoclassical radial electric field (2) can be approximated by the normalised ion pressure gradient $\nabla p_{i} / Z e n_{i}$ if the toroidal velocity is small. It was shown that in AUG this approximation fits the values of the radial electric field measured with CXRS in the plasma edge in typical H-mode conditions [13].

The I-phase has been described as an interplay between the turbulence and turbulent zonal flows that creates limit-cycle oscillations (LCO) [14] or as a relaxation 
of the mean radial electric field after a bifurcation of the poloidal flow to H-mode value [15. Some theoretical models include both the zonal flow and the mean shear flow [16] and maintain the primordial role of the zonal flow in the L-H transition. From the theoretical point of view LCO with different causality are possible, creating on the phase plane $\delta n_{e} / n_{e}-E_{r}$ cycles in clockwise or in counter-clockwise directions. Historically first it has been observed on AUG that there is a phase shift during I-phases in low-density plasmas [3]. In this case no modulation in the electron pressure gradient has been detected, as a consequence it has been supposed that the feeding mechanism of the I-phase is the predator-prey interplay between the turbulence and the zonal flows. On the contrary from EAST tokamak experiments it has been reported that there is no phase shift [9].

In general it is challenging to measure $E_{r}$, density, temperature and turbulence dynamics on a fast time scale. Therefore the mechanisms of the I-phase are difficult to determine from experiments. The experimental evidence of the presence of a zonal flow generally consists in the difference between the neoclassical and the total $\mathbf{E}_{\mathbf{r}} \times \mathbf{B}$ flow and has been reported at several machines [3, 16. However, by means of CXRS measurements of the perpendicular velocity and of the ion pressure in AUG, it was found recently that in some I-phases the background $E_{r}^{\text {neo }}$ dominates and oscillates in phase with the $\mathbf{E}_{\mathbf{r}} \times \mathbf{B}$ velocity and the turbulence level [17] within the existing time resolution of $100 \mu \mathrm{s}$. From this fact it has been deduced that zonal flow does not play a significant role in the L-H transition. At the same time on AUG it was shown that the signatures of the LCO during the I-phase correspond to the type III ELM relaxation [10]. In [8] it is also shown that the LCO occur without zonal flows.

Thus far, I-phases with and without zonal flow contribution have been reported and LCO phase space trajectories in both clockwise and counter-clockwise directions have been observed at different devices. The aim of the present study is to provide a better understanding of the turbulence, $\mathbf{E}_{\mathbf{r}} \times \mathbf{B}$ velocity shear and electron pressure gradient dynamics during the $\mathrm{L}-\mathrm{H}$ transition. In this contribution we investigate the turbulence behaviour using density fluctuation measurements from an ultra-fast swept reflectometer (UFSR) at AUG. This diagnostic is able to measure the electron density radial profile together with the turbulence level with a time resolution of the order of microseconds. The simultaneous measurement allows to localise and correlate density and density gradient perturbations at different radial positions. The normalised electron pressure gradient

$$
E_{r 0}=\frac{\nabla p_{e}}{Z e n_{e}}=\frac{\nabla T_{e}}{Z e}+\frac{T_{e} \nabla n_{e}}{Z e n_{e}}
$$

can be taken as a proxy for the normalised ion pressure gradient for high collisionality when $T_{i}=T_{e}$ can be assumed. However, this assumption is maybe a little too strong for the edge region where the equipartition time may be as large as a few milliseconds. Nevertheless, the measurements of the electron dynamics have the advantage of a better time resolution than one could achieve using the correct ion temperature and density. Therefore in this work the UFSR density measurements with $1 \mu$ s temporal resolution 
are combined with the ECE electron temperature data averaged over $100 \mu \mathrm{s}$ in order to follow the dynamics of the normalised electron pressure gradient. The work is focused on the fast dynamics of the second term in (3). A Doppler reflectometer system with several fixed frequency channels provides fast measurements of the $\mathbf{E}_{\mathbf{r}} \times \mathbf{B}$ velocity. The difference or the agreement between the normalised electron pressure gradient $E_{r 0}$ and $E_{r}$ independently measured by Doppler reflectometer can give some clues to describe the I-phase. A brief description of the ultra-fast swept reflectometer and the Doppler reflectometer system as well as of the methods of data analysis is given in the section 2. Section 3 is dedicated to the density, electrical field and normalised electron pressure gradient evolution during L-H transitions and I-phases with different plasma conditions. Finally, a discussion gives a possible explanation of the observed phenomena in the section 4 .

\section{Methods}

The tokamak ASDEX Upgrade has a set of techniques measuring the electron density: Interferometry, Vertical Thomson Scattering, Lithium Beam Emission Spectroscopy (LiBES), Ultra-Fast-Swept Reflectometry [18], FM-CW profile reflectometry [19], as well as Doppler backscattering reflectometry (DR) with several fixed frequency channels measuring the $E_{r} \times B$ flow velocity and the density fluctuations level [20]. The first two provide a temporal resolution of $300 \mu \mathrm{s}$ and $3 \mathrm{~ms}$, respectively. The Li-BES electron density profiles are measured with a high temporal resolution, but the radial access is limited to the edge and the scrape-off layer (SOL) with decreasing sensitivity towards the plasma core [21]. The recent transfer of the UFSR from Tore Supra to AUG has provided electron density measurements with a time resolution of $1 \mu$ s (less than the characteristic turbulence time scale of a few microseconds). The system consists of $\mathrm{V}$ and $\mathrm{W}$ band (50-105 GHz) frequency sweeping reflectometers [18]. The emitting and receiving bistatic antennas are installed on the mid plane of AUG at the low-field side with a line of sight in radial direction towards the centre of the plasma. The frequency bands of the probing waves are chosen such that the signal propagates from the lowfield side into the plasma until it is reflected at the cut-off layer with the X-mode cut-off frequency

$$
\omega_{ \pm}=\frac{\Omega_{c e}}{2}\left(1 \pm \sqrt{1+\frac{4 \omega_{p}^{2}\left(n_{e}\right)}{\Omega_{c e}^{2}}}\right)
$$

where $\omega_{p}^{2}=n_{e} e^{2} / \epsilon_{0} m_{e}$ and $\Omega_{c e}=e B / m_{e}$, with the electron density $n_{e}$, electron mass $m_{e}$, magnetic field strength $B$. The X-mode polarisation provides a large radial access from the very edge to the centre of the plasma for densities up to $5 \cdot 10^{19} \mathrm{~m}^{-3}$, reduced to the edge when the density is increased.

Using a linear frequency sweep and an iterative Bottollier-Curtet procedure [22] a density profile can be obtained. In order to detect the first cut-off frequency the associated amplitude jump of $10-20 \mathrm{~dB}$ is used. To detect this jump automatically the 
amplitude of the signal which is narrowly filtered around the beat frequency is smoothed and derived twice. The first and the second derivatives provide a precise localisation of the first cut-off frequency except of the cases of high density $\left(>8 \cdot 10^{19} \mathrm{~m}^{-3}\right)$ when the reflection from the lower cut-off $\omega_{-}$increases the amplitude level before the first cut-off of the upper branch $\omega_{+}$is reached. The accuracy of this method allows a milimeter precision in the density profile reconstruction.

The turbulent plasma density fluctuations can be extracted from the fluctuations of the reflected signal [23]. The heterodyne receiver and IQ detection assure a separation of phase and amplitude of the reflected signal with a signal to noise ratio of about $40 \mathrm{~dB}$, sufficient for fluctuation measurements. Fast and repetitive sweeps of $1 \mu \mathrm{s}$ with $0.25 \mu \mathrm{s}$ dead time between sweeps provide an equivalent sampling rate of $800 \mathrm{kHz}$ at a given probing frequency and allow to calculate radial density fluctuation profiles, frequency and wavenumber spectra.

In the case of low-level turbulence and O-mode polarization, the phase fluctuation wavenumber spectrum is directly proportional to the density fluctuation spectrum [24]. This concept has been extended to the $\mathrm{X}$-mode by introducing a radial wavenumber $\left(k_{r}\right)$ dependent transfer function, which needs to be found for each radial position and time using a 1D full-wave simulation [25]. Experimental density and magnetic field profiles, and predicted radial fluctuation wavenumber spectra are used as input and the simulation produces the signal phase fluctuation spectra as output. The ratio of input and output spectra gives the transfer function:

$$
T\left(k_{r}, R, t\right)=\frac{S_{\delta n}\left(k_{r}, R, t\right)}{S_{\delta \Phi}\left(k_{r}, R, t\right)} .
$$

A closed-loop minimizing method [26] is applied to reduce the difference between the numerical and measured phase spectra. If they are close enough, the input at this iteration is assumed to correspond to the real density fluctuation spectra and to be the unique solution, otherwise the current transfer function is applied to the experimental phase spectra and the result is used as input for the next iteration. The difference between the output and measurements is taken as an error bar estimation and the looping terminates when the error becomes sufficiently small, of the order of a few percent.

Due to the Bragg condition it is possible to get information about density fluctuations with wave numbers $k_{f}$ twice that of the probing wave $\left(k_{f}=2 k_{r}\right)$, here the probing wavenumber varies in the cut-off region from 0 to $10 \mathrm{~cm}^{-1}$. The lower $k_{r}$ limit depends on the sample window size used in the reconstruction, giving 1$2 \mathrm{~cm}^{-1}<k_{r}<10 \mathrm{~cm}^{-1}$ for the detected density fluctuations. This corresponds to turbulent scales in the range $0.1<k_{r} \rho_{s}<1$ for typical AUG conditions. By integrating the wavenumber spectra over $k_{f}$, a radial profile of the density fluctuation level $\delta n / n$ can be reconstructed [25]

$$
\left(\frac{\delta n}{n}\right)^{2}=\frac{1}{n_{c o}^{2}\left(k_{\max }-k_{\min }\right)} \int_{k_{\min }}^{k_{\max }} S_{\delta n}\left(k_{r}\right) d k_{r},
$$


where $n_{c o}$ is the density at the cut-off. To reduce the uncertainties, the method should be improved by including some constraining hypotheses on the transfer function (Eq. 5). During the iterations the $k_{r}$ input spectrum is constrained to have negative slope values. If the absolute spectral power starts to diverge and the corresponding turbulence level exceeds $100 \%$, the input spectra are devided by a constant to reset the input turbulence level to $10 \%$ in the edge. This corresponds to the case of a low turbulence level, when it is proved that $1 \mathrm{D}$ simulation provides an accurate estimation of the density fluctuation level [25]. Other restricting hypotheses could be made via $2 \mathrm{D}$ full-wave simulations and the corresponding work is ongoing [27]. The optimal solution would be to find an analytical expression for the transfer function depending only on plasma parameters. By now the limiting conditions on the spectral power and on the slope of the $k_{r}$ spectrum are applied and, in order to reduce the calculation time, the same initial slopes are put in for few milliseconds of consecutive sweeps.

The Doppler reflectometry backscattering system provides measurements of density fluctuation characteristics with good spatial resolution at selected perpendicular wavenumbers. The probing wave is launched in oblique incidence with respect to the normal vector to the surfaces of constant refractive index. In this case only the signal backscattered on density fluctuations close to the turning point is detected. The radial position of the cut-off and the wavenumber for the probing wave are found from the 3D beam tracing code TORBEAM [28] simulating the propagation of a Gaussian beam in a stationary plasma.Due to the turbulent structures velocity perpendicular to the magnetic field lines, the received signal is Doppler shifted by $\omega=\mathbf{k} \cdot \mathbf{v}=k_{\|} v_{\|}+k_{\perp} v_{\perp}$. The probing beam is launched perpendicular to the magnetic field so $k_{\|}$and thus the parallel component contribution are small. Close to the cut-off, the perpendicular component $k_{\perp}$ is mainly orthoradial so the radial component is also expected to be small, except during relaxations of the pedestal where strong radial perturbatoins before the cut-off can contribute to the signal, as it may happen during ELMs or expulsion of filaments. Otherwise the extracted velocity $v_{\perp}=\omega / k_{\perp}$ is assumed to correspond to the perpendicular velocity of the density fluctuations in the laboratory frame, $v=v_{E \times B}+v_{p h}$, where $v_{E \times B}$ is the $\mathbf{E} \times \mathbf{B}$ velocity and $v_{p h}$ is the phase velocity of the fluctuations. It has been shown in several experiments that the phase velocity is small compared to the $\mathbf{E}_{\mathbf{r}} \times \mathbf{B}$ velocity at the edge of a stationary plasma [29]. Hence the Doppler shift is used as an approximation for $\mathbf{E}_{\mathbf{r}} \times \mathbf{B}$ flow and thus of the radial electric field. Several Doppler systems installed on AUG have in total six channels, 4 operating in $\mathrm{V}$-band frequency range and 2 in $\mathrm{W}$-band. To extract the Doppler frequency on a fast time scale the multiple signal classification (MUSIC) algorithm is used [30], with a typical window length of about 1.5 to $3 \mu \mathrm{s}$, together with the spectrogram analysis (fast Fourier transform on short sliding windows of about $10 \mu \mathrm{s}$ ). The evaluation of the velocity is done only for times when the signal amplitude exceeds a threshold set at about three times the noise level. This limits $E_{r}$ measurements to plasmas where turbulence is not suppressed too strongly. 


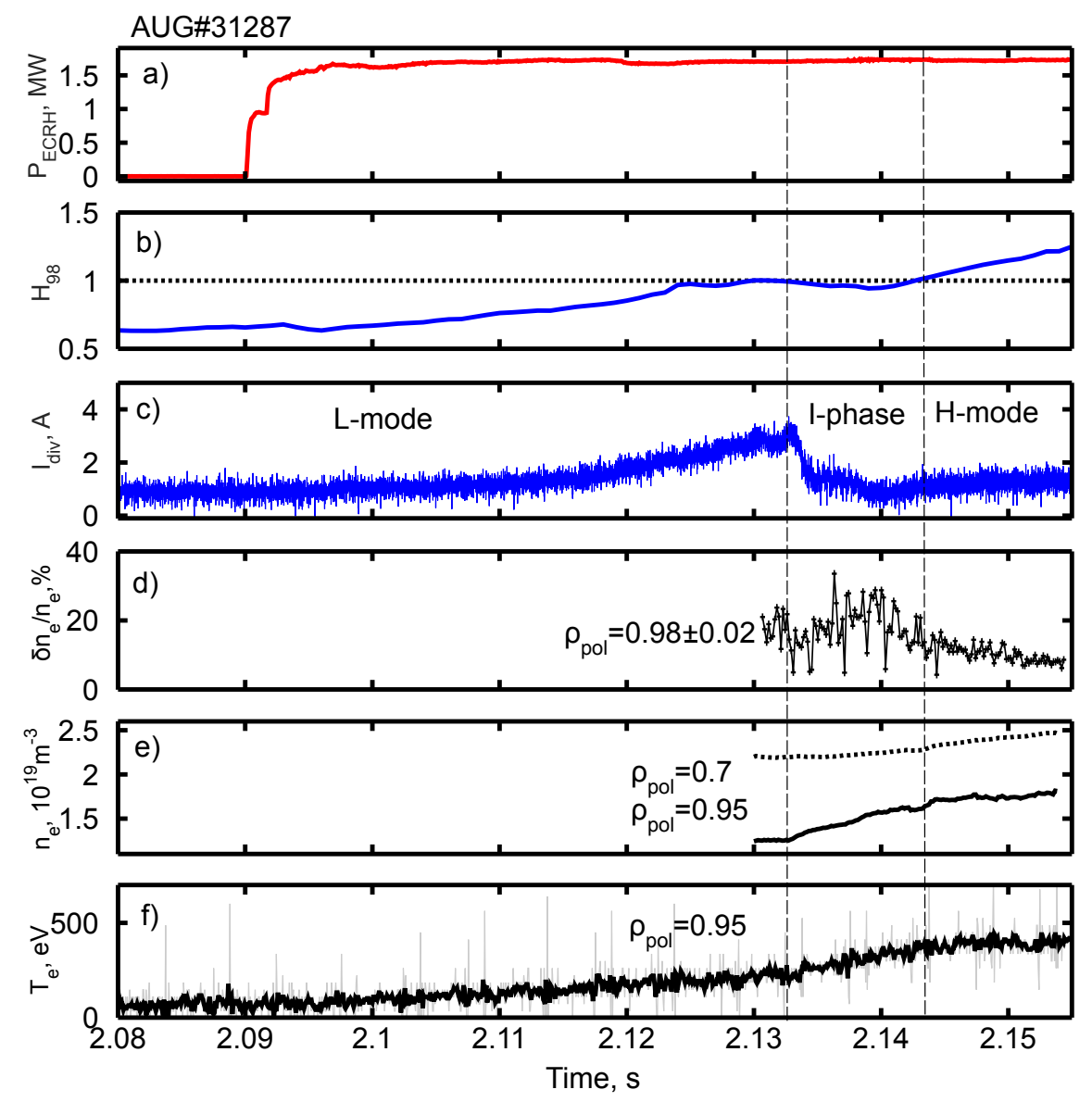

Figure 1. Low-density L-H transition: a) electron-cyclotron heating power, b) Hfactor, c) divertor current, d) density fluctuation level at $\rho_{p o l}=0.98$, e) pedestal top (solid) and core (points) density from UFSR, f) pedestal top temperature from ECE

\section{Experimental results}

The results that are presented here have been obtained by analysing a series of discharges with both L and H-mode phases performed in AUG. The D or He plasma was heated using electron cyclotron resonance heating $(\mathrm{ECRH})$ at $140 \mathrm{GHz}$, which corresponds to core heating at a magnetic field of 2.40-2.56 $\mathrm{T}$ used in these discharges. The power step amplitude has been chosen for each density to be slightly above the threshold for an L-H transition (Fig. 1 17), using the power threshold scaling from [31]. Different central plasma densities from 3 to $7 \cdot 10^{19} \mathrm{~m}^{-3}$ have been chosen in order to check the universality of observed phenomena. Fig. 1 shows the time trace for the H-factor $H_{98}=\tau_{E} / \tau_{I P B 98(y, 2)}(\mathrm{b})$, where $\tau_{E}$ is the energy confinement time and $\tau_{I P B 98(y, 2)}$ is the scaling based on the set of ELMy H-mode data from different tokamaks [32], indicating the confinement improvement in H-mode for the shot \#31287 with core line averaged density of $3 \cdot 10^{19} \mathrm{~m}^{-3}$. The $\mathrm{L}-\mathrm{H}$ transition can be conveniently detected on the divertor current indicating the particles confinement change (Fig. 1 $\mathrm{c}$ ) and on the pedestal top 


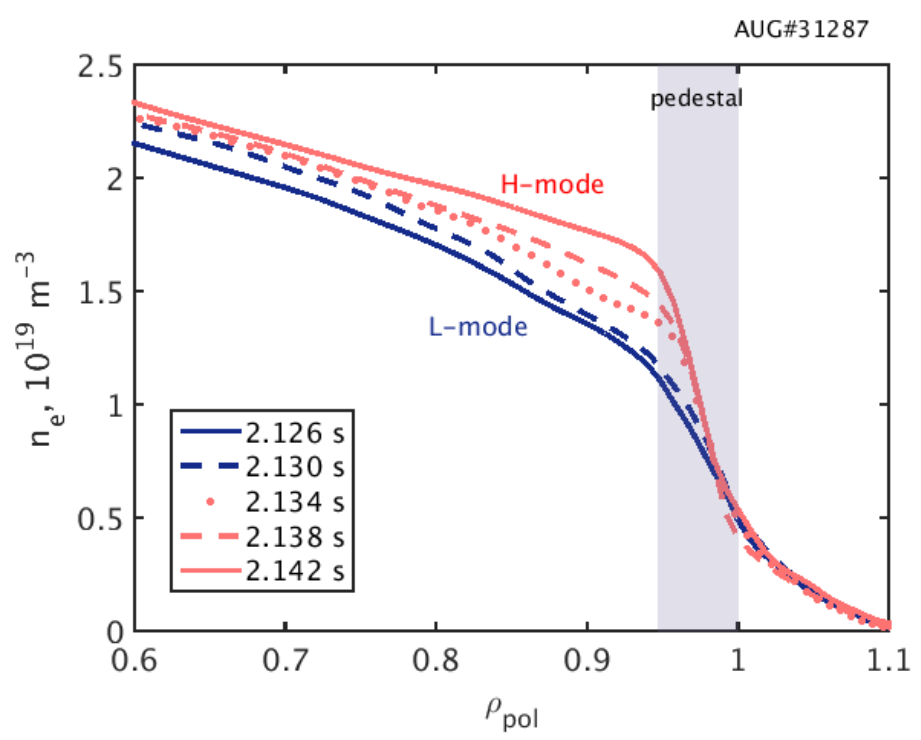

Figure 2. Density profile evolution during the L-H transition, L-mode at 2.126$2.130 \mathrm{~s}$, I-phase at 2.134-2.138 s, H-mode after $2.142 \mathrm{~s}$, profiles averaged over $1 \mathrm{~ms}$

density (at a normalised radius of $\rho_{\text {pol }}=0.95$ ) that has a rapid grow (Fig. 1, solid line) while the gas puff is kept constant. The core density builds up slower (Fig. 1, dashed line), so the edge density gradient is significantly increased as well as the edge temperature gradient. The evolution of the electron density profile can be seen in Fig. 2. the profile builds up inside $\rho_{\text {pol }}=0.99$ from shallow in the L-mode $(2.126-2.132 \mathrm{~s})$ to steep in the H-mode (after $2.142 \mathrm{~s}$ ). Every L-H transition on AUG typically passes through an I-phase. Although the plasma density and temperature oscillations happen during the whole I-phase, the strong density gradient appears after the first milliseconds of the I-phase. At the same time the turbulence level strongly oscillates (Fig. 11d) from 20-30 \% to small values of about $10 \%$ that indicate the building of the transport barrier in the very edge of the plasma, characteristic for the H-mode behaviour.

The L-H transition occurs 20-60 ms after the heating start as it is seen around $2.131 \mathrm{~s}$ in the drop of the outer divertor current (Fig. 1. $)$. The average turbulence level in the edge decreases from $20 \%$ in L-mode to $10 \%$ in H-mode (Fig.11d), which is a typical behaviour for AUG H-mode [33]. As the UFSR has limited acquisition memory, in the high sweeping rate mode the system could acquire only $30 \mathrm{~ms}$ during the AUG campaign 2014 and $250 \mathrm{~ms}$ in 2015. For this reason the trigger for measurements has been set a few milliseconds prior to the expected $\mathrm{L}-\mathrm{H}$ transition, so the density fluctuation level (Fig. 11) and the precise density evolution (Fig. 17) are typically available only for a part of the discharge. Immediatly after the reduction of particle transport to the outer divertor, high-frequency modes appear in the frequency spectra of density fluctuations. The modes are characterised by narrow frequency bands and high coherence within a broad radial extent in the plasma edge. The description of the edge coherent modes is given in subsection 3.1. The detailed analysis of the L-H transition and of the I-phase 

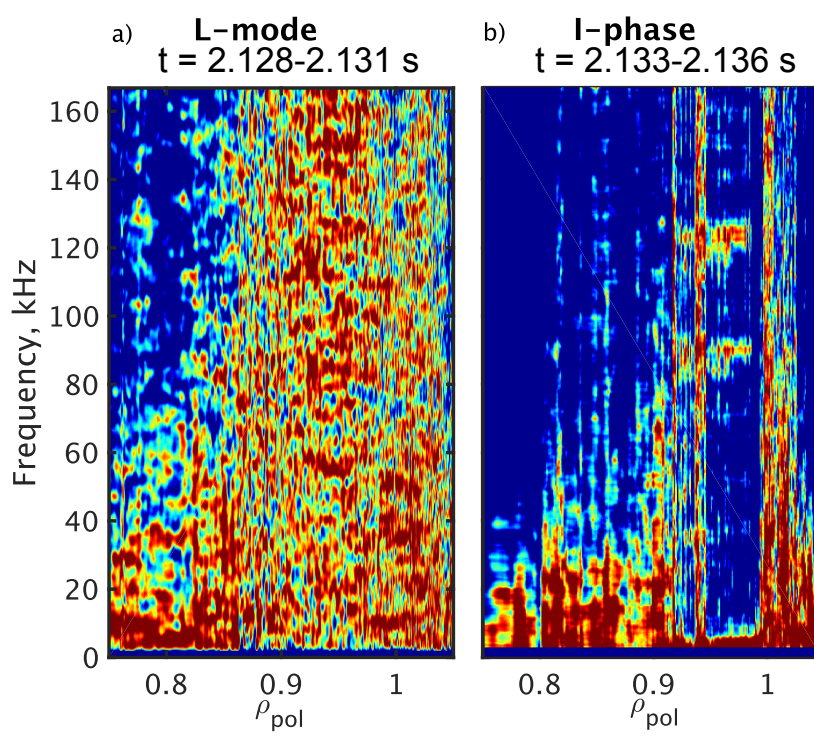

$\begin{aligned} \text { c) } & \text { H-mode AUG\#31287 } \\ t= & 2.150-2.153 \mathrm{~s}\end{aligned}$
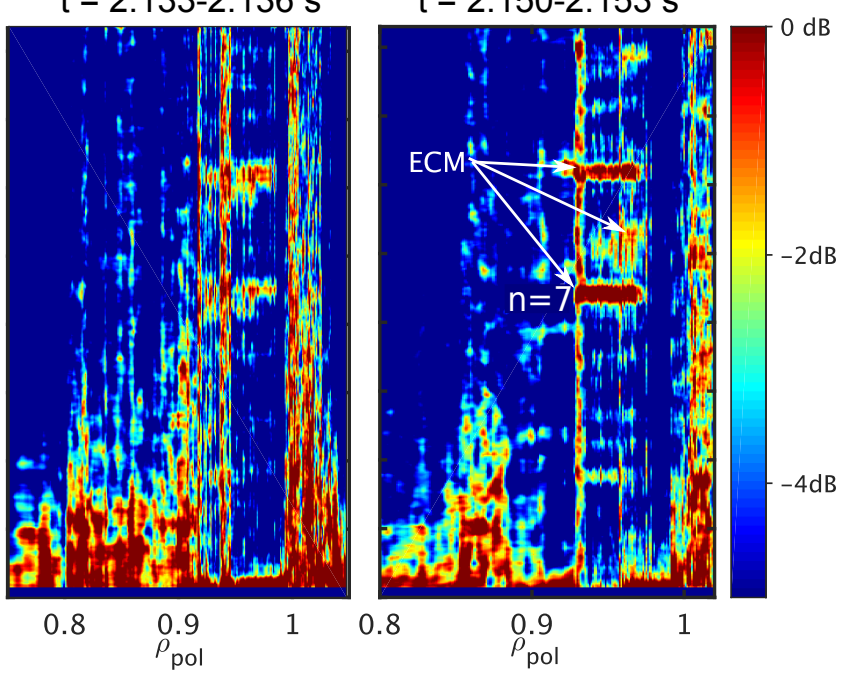

Figure 3. Density fluctuation frequency power spectra a) in L-mode, b) during I-phase and c) in H-mode from the UFSR

is presented in subsection 3.2. The comparison of $\mathrm{L}$ and H-mode density fluctuation characteristics is discussed in subsection 3.3.

\subsection{Edge coherent modes}

To characterise the turbulence present in L- and H-mode the frequency spectra have been obtained for the complex signal for each of the 2000 probing frequencies of the UFSR from 50 to $100 \mathrm{GHz}$, corresponding to 2000 radial positions of the cut-off, and interpolated on the normalized radius using an average density profile. This method provides a high radial resolution for the precise localisation of plasma modes, self consistent with the density profile. Figure 3 depicts the frequency power spectra of the density fluctuation. Three time windows of $3 \mathrm{~ms}$ each have been chosen to describe the spectra modification in L-mode (a), H-mode (c) and during the I-phase (b). In the pedestal region, $0.90<\rho_{\text {pol }}<1$, during the I-phase the fluctuation amplitude decreases and the spectra become narrower compared to the L-mode broadband turbulence.

Edge coherent modes (ECM) appear directly at the transition from the L-mode to the I-phase on the top of the pedestal at $0.92<\rho_{\text {pol }}<0.98$ around $125 \mathrm{kHz}$ for the lowdensity discharge \#31287. The same kind of modes have been observed with the UFSR across L-H transitions and in quiescent $\mathrm{H}$-mode discharges, at frequencies in the range of 60-150 kHz without any obvious dependence on the plasma parameters. Although the appearance of the modes is connected with the L-H transition, it is unknown whether they contribute to the mechanisms of the transition.

From swept reflectometry the radial localisation and extent of this coherent mode 
is deduced to be $0.92<\rho_{\text {pol }}<0.99$. The radial movement of the profile during the FFT time window can introduce an additional broadening of the mode in the spectra of about $\Delta \rho_{\text {pol }}=0.02$. Toroidal mode numbers were deduced from the Mirnov coils. They vary between $n=-3$ and -11 for the lowest to the uppermost branch (the negative sign corresponds to the electron diamagnetic direction). As the modes develop with the increase of the pressure gradient they might be related to a kinetic ballooning instability [34].

The analysis of the density fluctuations though becomes complicated as the coherent modes dominate the turbulent spectrum. In order to distinguish density fluctuations due to these modes and broadband turbulence, a low-pass filter $f<50 \mathrm{kHz}$, excluding the ECM, has been applied to the UFSR signal phase. The density fluctuation profile shape is unchanged after filtering, the absolute level of density fuctuation decrease proportionally to the filter width, hence the ECM do not contribute to the selected $k_{r}$ range from 2 to $20 \mathrm{~cm}^{-1}$. That might mean that the edge coherent modes have a small $k_{r}<2 \mathrm{~cm}^{-1}$. It reveals that the closed-loop method is not able to follow the edge coherent modes dynamics. In the H-mode the frequency spectra stay approximately constant, as well as the integrated turbulence level. Thus the main interest for the L-H transition study is a detailed description of the I-phase.

\subsection{I-phase}

During this study, I-phases for various plasma conditions were documented and the contribution of the background flow to the total $\mathbf{E}_{\mathbf{r}} \times \mathbf{B}$ flow has been analysed. During the first milliseconds after the divertor current drop, the I-phase has been observed for each discharge from the experimental series. Figure 4 shows an example of the I-phase starting at $3.117 \mathrm{~s}$. The upper plot demonstrates the evolution of the normalised electron pressure gradient $E_{r 0}$ at different radial positions. The pressure has been calculated from UFSR density data with $1 \mu$ s resolution averaged over $10 \mu$ s and from the ECE temperature measurements averaged over $100 \mu \mathrm{s}$. A correction of the shine-through effect has been included in the ECE temperature profiles reconstruction. The average edge electron density starts to grow continuously from the beginning of the I-phase until the first type-I ELM at $3.245 \mathrm{~s}$ (Fig. 4p). Strong oscillations of the density and of $E_{r 0}$ are visible on both plots. Figure 4 c shows a spectrogram of $E_{r 0}$ at $\rho_{\text {pol }}=0.98$ calculated with sliding FFT over time windows of $10 \mathrm{~ms}$. The $\mathrm{kHz}$ oscillations can be observed with the frequency changing from $2 \mathrm{kHz}$ in the beginning of the I-phase to $1 \mathrm{kHz}$ in the established I-phase. The frequency evolution agrees with the scaling of the I-phase oscillation period with the density observed previously [10]. The modulation of $E_{r 0}$ is localised in the pedestal region, $0.94<\rho_{p o l}<1$. During the I-phase the average normalised electron pressure gradient continuously deepens around $\rho_{\text {pol }}=0.98$ until the typical H-mode transition value of radial electric field on AUG below $-15 \mathrm{kV} / \mathrm{m}$ [31] is reached. On top of this evolution, $E_{r 0}$ undergoes highly localised $\mathrm{kHz}$ oscillations.

After a heating pulse significantly exceeding the power threshold for an L-H 


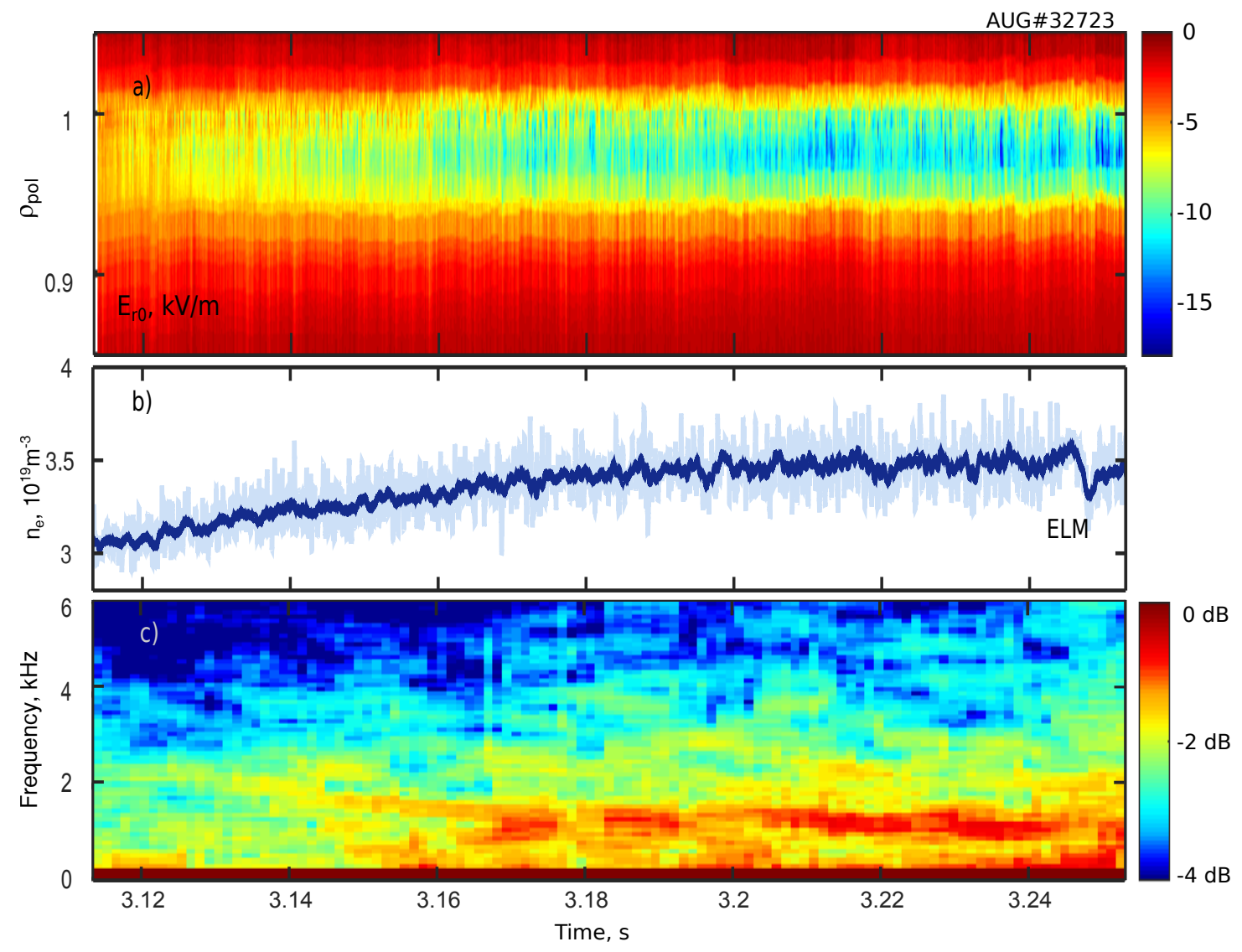

Figure 4. a) Normalised electron pressure gradient during the I-phase, b) edge electron density during the I-phase (3.117-3.245 s), c) spectrogram of $E_{r 0}$ at $\rho_{\text {pol }}=0.98$ where the I-phase oscillations are observed with frequency from 2 to $1 \mathrm{kHz}$

transition, the I-phase is typically short. The discharges \#31287, \#32723 and \#32944 have been chosen as representative cases of various plasma conditions. The shot \#31287 represents a low-density case, with the core density of about $4 \cdot 10^{19} \mathrm{~m}^{-3}$ and a fast L-H transition with a short I-phase duration of $10 \mathrm{~ms}$ (Fig. 1). The shot \#32723 shows a longer I-phase of $130 \mathrm{~ms}$ in a He plasma with high central density of $7 \cdot 10^{19} \mathrm{~m}^{-3}$ (Fig. 4). Shot \#32944 is a medium-density case $\left(4.5 \cdot 10^{19} \mathrm{~m}^{-3}\right)$ with an I-phase lasting $20 \mathrm{~ms}$. The behaviour of density gradient and density fluctuations appear to be similar for the three indicated cases. The frequency of I-phase oscillations is lower for high density discharges [10], so the analysis of this case provides better statistics with a given time resolution and thus the focus will be on the discharge \#32723.

In AUG the I-phase can typically be identified in a poloidal Mirnov coil signal $\partial B_{\theta} / \partial t$ as low $\mathrm{kHz}$ range oscillations as it is shown in the zoom on the first Iphase oscillations (Fig. 5e) for the Mirnov coil C09-23 situated close to the X-point. The turbulence level periodic suppression is visible in the amplitude of the Doppler reflectometer signal after $t=3.1171 \mathrm{~s}$ (Fig. 5d) and synchronously in the density fluctuation level measured by the UFSR at about $\rho_{\text {pol }}=0.96-1$ (Fig. 5a,b). Similar 


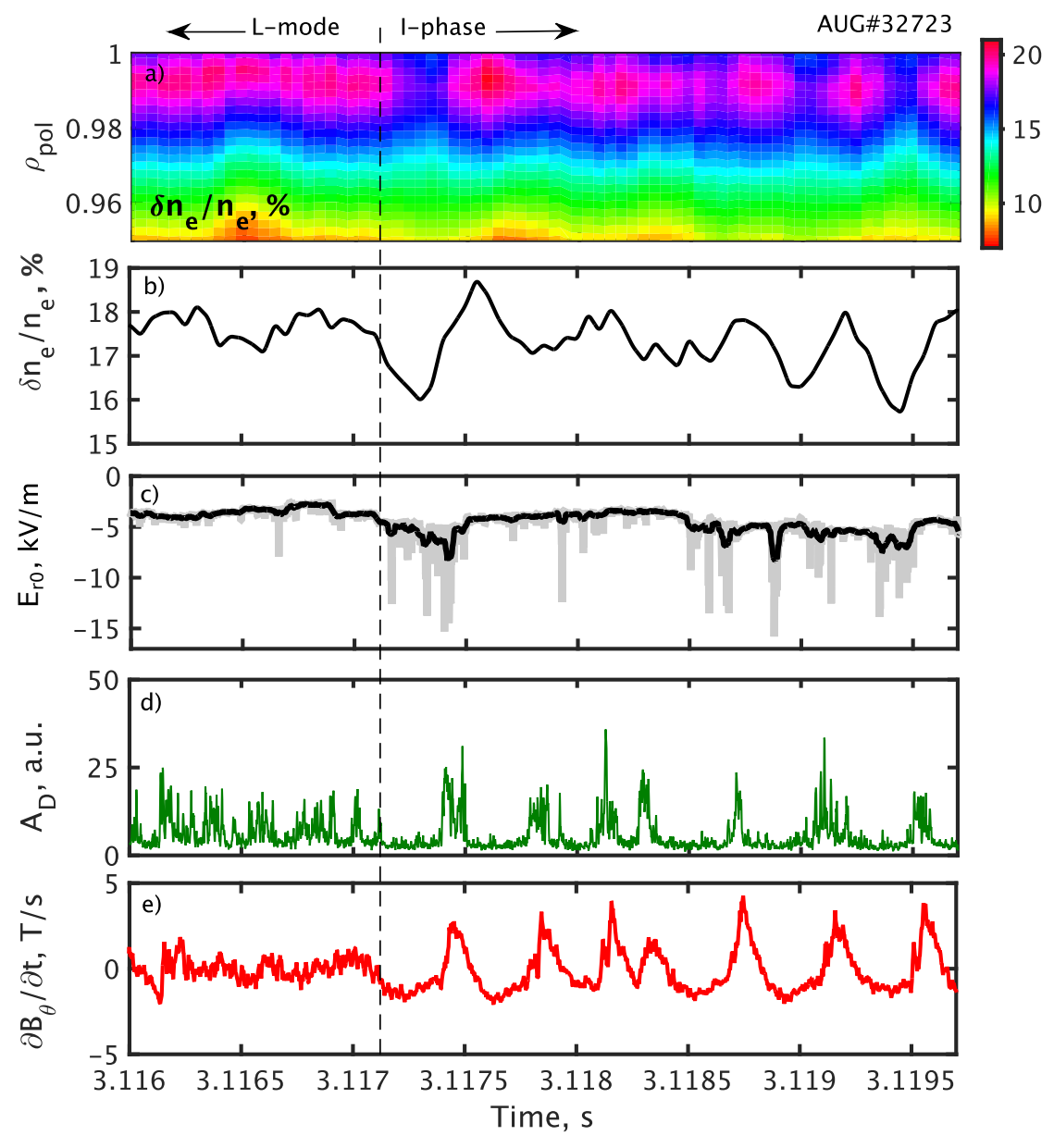

Figure 5. a) Density fluctuation level in the plasma edge, b) at $\rho_{\text {pol }}=0.99$, c) normalised electron pressure gradient $E_{r 0}$ at $\rho_{p o l}=0.99$, d) Doppler reflectometer signal amplitude for $\mathrm{F}=80 \mathrm{GHz}$, e) Mirnov coil signal during the first oscillations of the I-phase

bursts have been found with a high temporal resolution in the normalised electron pressure gradient $\left(E_{r 0}\right)$ evolution in the pedestal region. The close-up on the oscillations shows that during one period of the I-phase $E_{r 0}$ (Fig. 5 c in grey with the time resolution of $10 \mu$ s and in black with the average of $100 \mu$ s for density) changes by about $30 \%$, the crash to the lower absolute level (as at $t=3.1174 \mathrm{~s}$ or $t=3.1195 \mathrm{~s}$ ) being synchronised with the magnetic signal $S_{m a g}=\partial B_{\theta} / \partial t$ bursts and the increase of density fluctuation level $\delta n_{e} / n_{e}$. Although the first oscillations of the I-phase $(t=3.1175-3.119 \mathrm{~s})$ have irregular character, during the most part of the I-phase the deep negative values of $E_{r 0}$ appear between the turbulence bursts and the crash of $E_{r 0}$ happens together with the turbulence increase. Before the very first I-phase burst at $t=3.1174 \mathrm{~s}$ the turbulence level drops and the $E_{r 0}$ minimum reaches the typical threshold of the radial electric field for $\mathrm{L}-\mathrm{H}$ transition, this stage will need further investigation to understand the triggering 

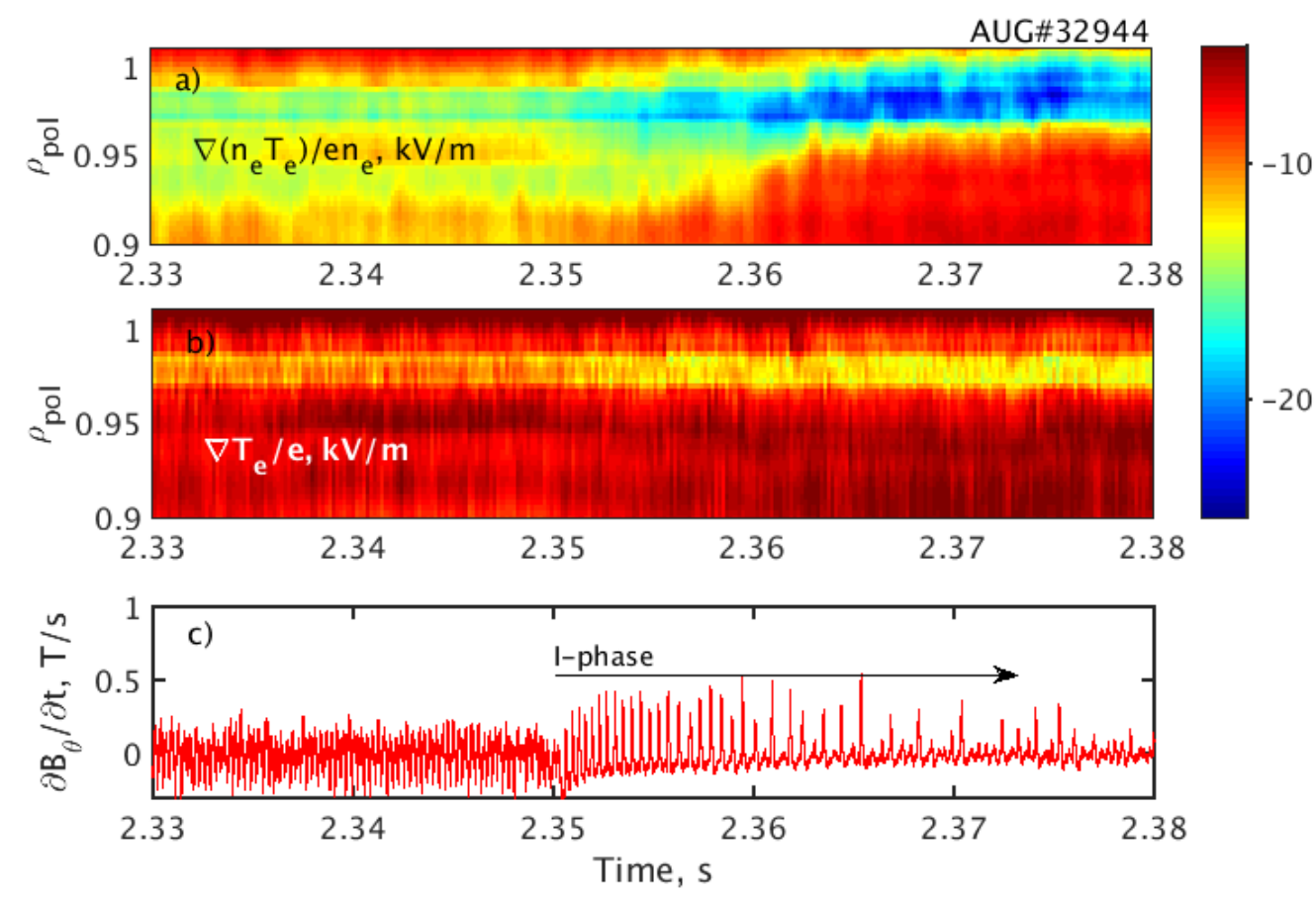

Figure 6. Nomalised electron pressure gradient a), $\nabla T_{e} / e$ contribution b) and magnetic signal $\partial B_{\theta} / \partial t \mathrm{c}$ ) during the I-phase $t=2.35-2.37 \mathrm{~s}$

mechanisms of the transition.

The medium density I-phase is shown in Fig. 6. The signal from the Mirnov coil defines the beginning of the I-phase at $2.35 \mathrm{~s}$. The contribution of the temperature gradient term to the pressure gradient is about $50 \%$. The temperature spatial resolution does not allow to follow the radial movement of the $E_{r 0}$ minimum, while the density gradient shifts the normalised electron pressure gradient minimum from $\rho_{\text {pol }}=0.96$ to 0.98 during the I-phase (Fig. 6a). Hence the precise measurement of the density and its gradient ensures a better understanding of the I-phase dynamics.

In order to follow the displacement of $E_{r 0}$ the radial correlation of $E_{r 0}$ with respect to $E_{r 0}$ at $\rho_{p o l}=0.98$ has been reconstructed (Fig. 7) for the time window $t=3.191-$ $3.194 \mathrm{~s}$. The maximum of the correlation function for each radial position is marked with a black cross. The correlation function is periodic in the time delay $\Delta t$ with the I-phase oscillation period of $1.3 \mathrm{kHz}$. The $E_{r 0}$ modulation propagates from the top of the pedestal to $\rho_{p o l}=0.98$. The crash of $E_{r 0}$ happens simultaneously within the given time resolution at all radial positions from $\rho_{\text {pol }}=0.98$ to the separatrix, which is also confirmed from different Doppler reflectometer channels by observing the same time evolution of the Doppler shift simultaneously or with a small delay for outer channels. At the separatrix $E_{r 0}$ modulation changes the phase by $\pi$, i.e. is anti-correlated with the modulation within the last closed magnetic surface. The time delay between inner radial positions is of the order of $30 \mu$ s and should be considered in the analysis of the relative phase. The correlation rapidly decreases to the noise level for $\rho_{\text {pol }}<0.96$ 


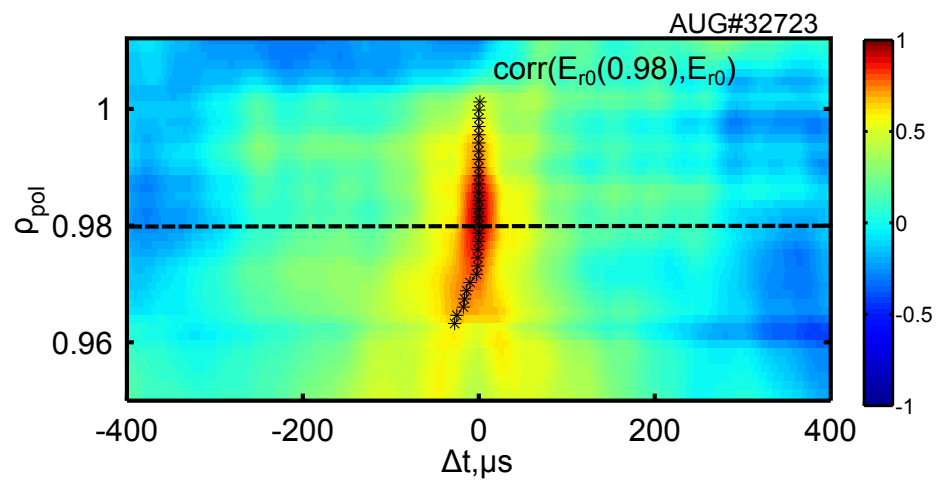

Figure 7. Correlations of the normalised electron pressure gradient $E_{r 0}$ at different radial positions and the propagation of its modulation inside the separatrix
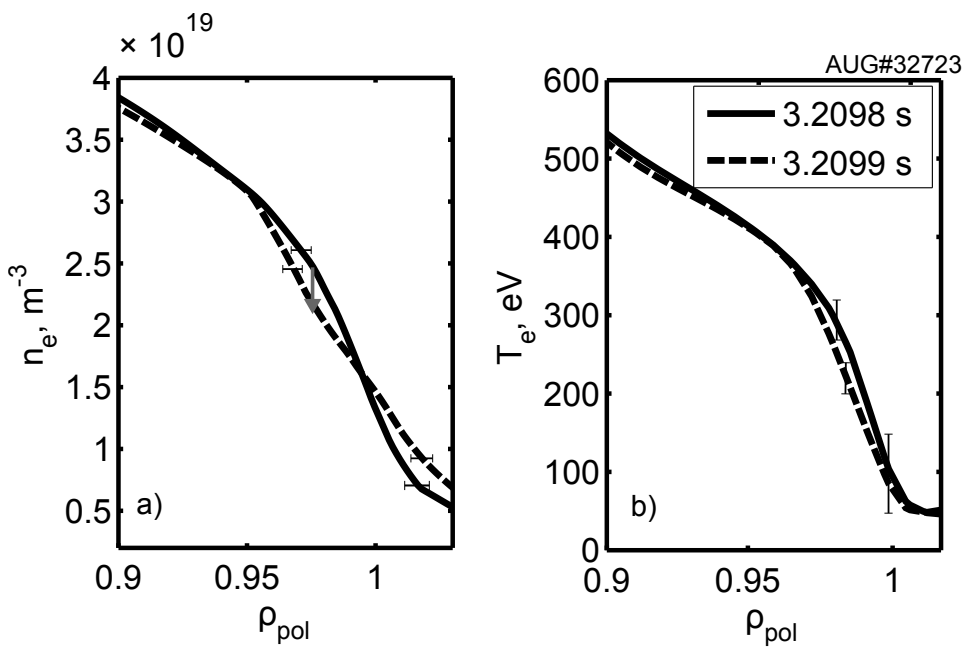

Figure 8. Example of density a) and temperature b) profile dynamics during a late I-phase burst

which means that the I-phase $E_{r}$ modulation is localised in the pedestal region. The anticorrelation inside and outside the separatrix has the usual characteristics of a crash of the edge density gradient which starts at the pedestal top.

The evolution of the pressure is accompanied by a significant change of the density profile. During one burst of the I-phase the density crashes inside the separatrix and grows outside (Fig. 8), which is similar to the behaviour during ELMs with a periodic outward particle expulsion. The pedestal top density changes by about $15-20 \%$, crashes in $100-200 \mu \mathrm{s}$ and rebuilds until the next crash during 300-1000 $\mu \mathrm{s}$. The density 


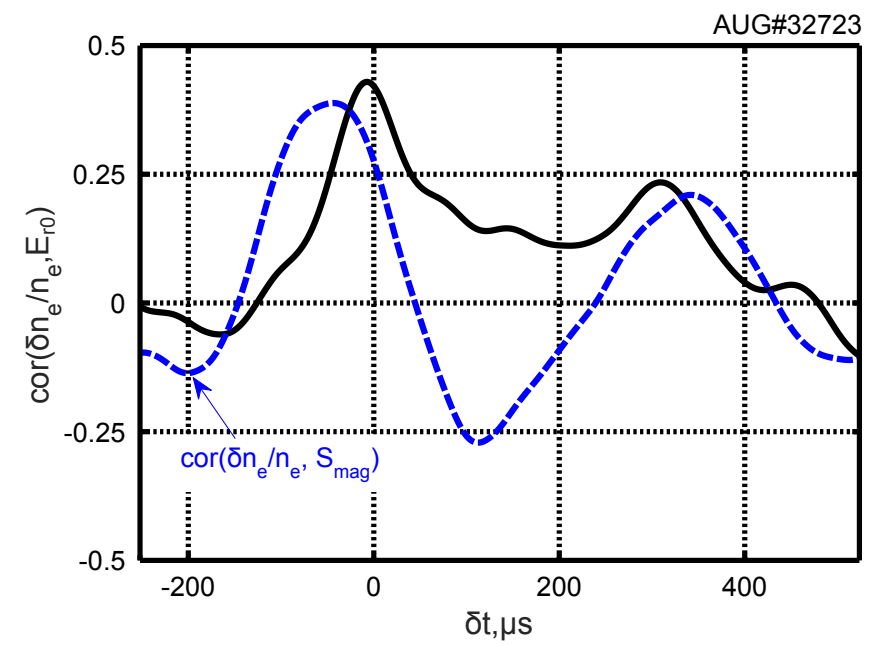

Figure 9. Correlation of the normilised electron pressure gradient $E_{r 0}$ and $\delta n_{e} / n_{e}$ (solid) and $\delta n_{e} / n_{e}$ with $\partial B_{\theta} / \partial t$ (dashed blue) at $\rho_{\text {pol }}=0.98$ for time window $\mathrm{t}=$ 3.19-3.20 s, AUG\#32723

evolution is taken into account for the Doppler measurement position. The pedestal top temperature oscillates as well with 5-10\% amplitude which is hardly distinguished within large error bars.

A long I-phase with many periods of oscillations ( $>10 \mathrm{~ms}$ ) can be devided in two parts: the early I-phase where the oscillation frequency changes significantly during several periods (Fig. 4 4 , $t<3.17 \mathrm{~s}$ ) and the established I-phase where it can be cosidered constant during at least ten periods. The period of the I-phase changes from $400 \mu \mathrm{s}$ in the early I-phase to $1 \mathrm{~ms}$ before the first ELM. The late or established I-phase in the medium to high density discharges can be characterised by the regularity of its bursts and by the zero phase shift between the turbulence and the normalised electron pressure gradient. Indeed, the cross-correlation function reconstructed between the density fluctuation level $\delta n_{e} / n_{e}$ and $E_{r 0}$ at $\rho_{p o l}=0.98$ for a time window of $10 \mathrm{~ms}$ in the established I-phase demonstrates the absence of the phase shift (Fig. 9, black solid) within a time resolution of $10 \mu \mathrm{s}$. This can be interpreted as the absence of predator-prey interaction between $E_{r 0}$ and the turbulence with radial wavenumbers of $1-10 \mathrm{~cm}^{-1}$. A small negative phase shift indicates that the fluctuation level increases during the strong $E_{r 0}$ phase because the steep pressure gradient drives the edge instabilities. Note that the oscillations in the early I-phase are not regular and their frequency evolves too fast to allow a reconstruction of the correlation function over a time window with 10-20 oscillations.

Both $E_{r 0}$ and the turbulence level oscillations are slightly shifted with respect to the magnetic signal (Fig. 9, blue dashed) in the established I-phase. The shift of the correlation function of $\Delta t=50 \mu \mathrm{s}$ visible also in Fig. 5b,e is probably due to the location of the Mirnov coil close to the X-point compared to the mid-plane position of the UFSR. This shift indicates that the I-phase oscillations propagate from the X-point to the top of the tokamak from the low-field side. This behaviour is consistant with the magnetic 


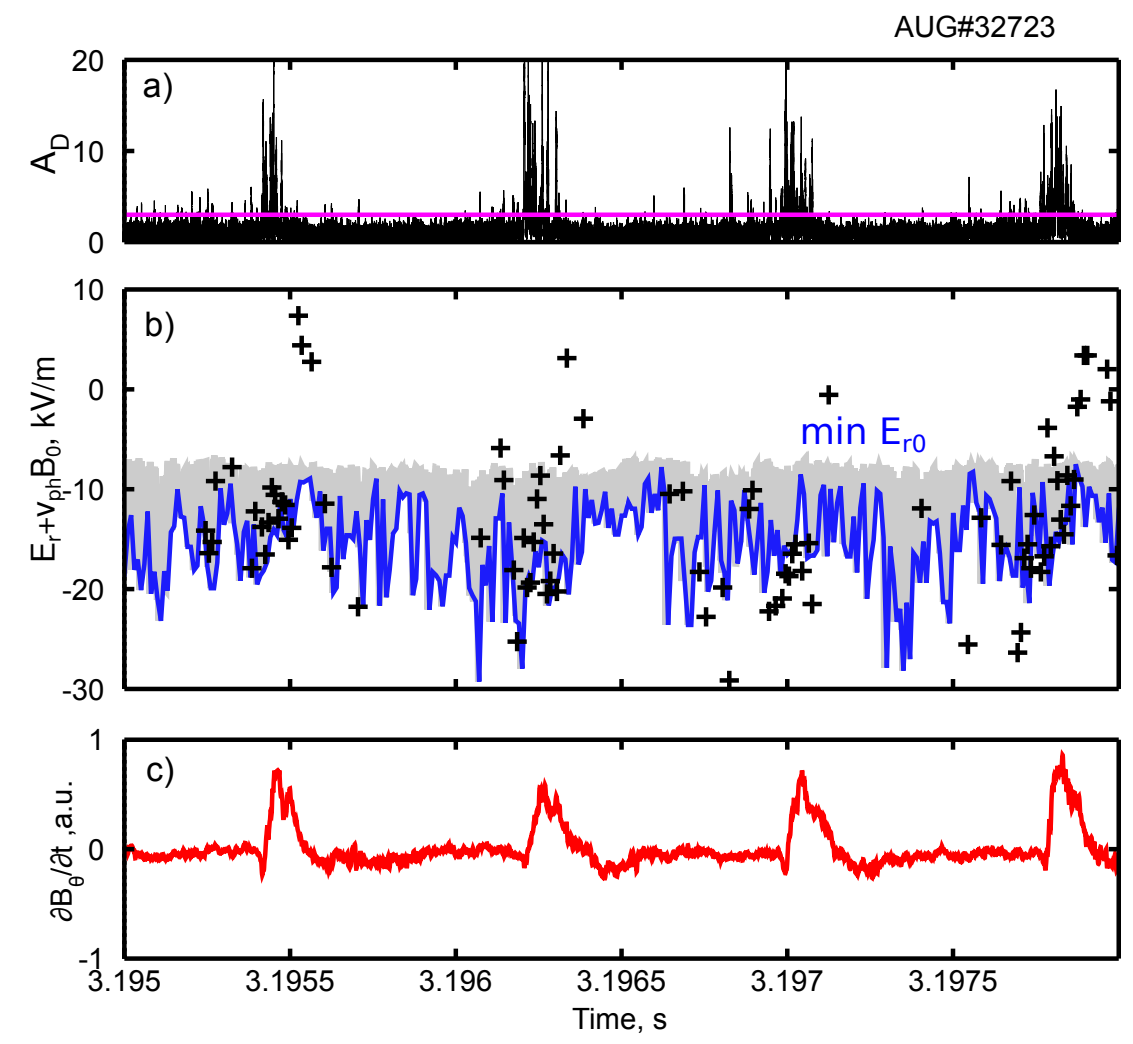

Figure 10. Doppler reflectometer signal amplitude at $\mathrm{F}=80 \mathrm{GHz}, \mathrm{X}$-mode a), estimation of $E_{r}$ minimum value from 3 channels of Doppler reflectometers (crosses), the $E_{r 0}$ minimum (grey) and its minimum values inside windows of $10 \mu \mathrm{s}$ (blue) b), the Mirnov coil signal $\partial B_{\theta} / \partial t \mathrm{c}$ ) indicates the I-phase bursts

structure and the poloidal propagation of the I-phase oscillations discussed in [10].

The DR data from three fixed frequency channels $(80,85 \mathrm{GHz}$ X-mode and $55 \mathrm{GHz}$ O-mode) have been used for the comparison between the total $E_{r}$ and the neoclassical proxy $E_{r 0}$. Only the points with the signal amplitude $A_{D}$ (Fig. 10a, black line) higher than three times the noise level (Fig. 10a, magenta) were taken into account. The density profile evolution changes the position of DR measurement and makes all the channels scan the region of $0.96<\rho_{\text {pol }}<1$. The wavenumber calculated from the beam tracing changes by about $5-15 \%$. Therefore, in order to obtain the measurement from the position nearest to the $E_{r}$ well minimum, for each time window of $10 \mu$ s the minimum value from the 3 channels has been selected.

The result of this selective procedure is shown in Fig. 10b with crosses and is taken as an estimation of the $E_{r}$ minimum value. Note the Doppler measurement corresponds to the sum of three terms $E_{r}+\frac{u_{r} k_{r}}{k_{\perp}} B_{0}+v_{p h} B_{0}$, where the first term is dominant if the radial movement of plasma is excluded and the phase velocity $v_{p h}$ is considered small. In order to compare similar quantities, the $E_{r 0}$ minimum (grey line) was calculated for time windows of $10 \mu \mathrm{s}$ and the minimum value for each window is shown as the blue line. The fast oscillation of the normalised pressure gradient is probably due to the 

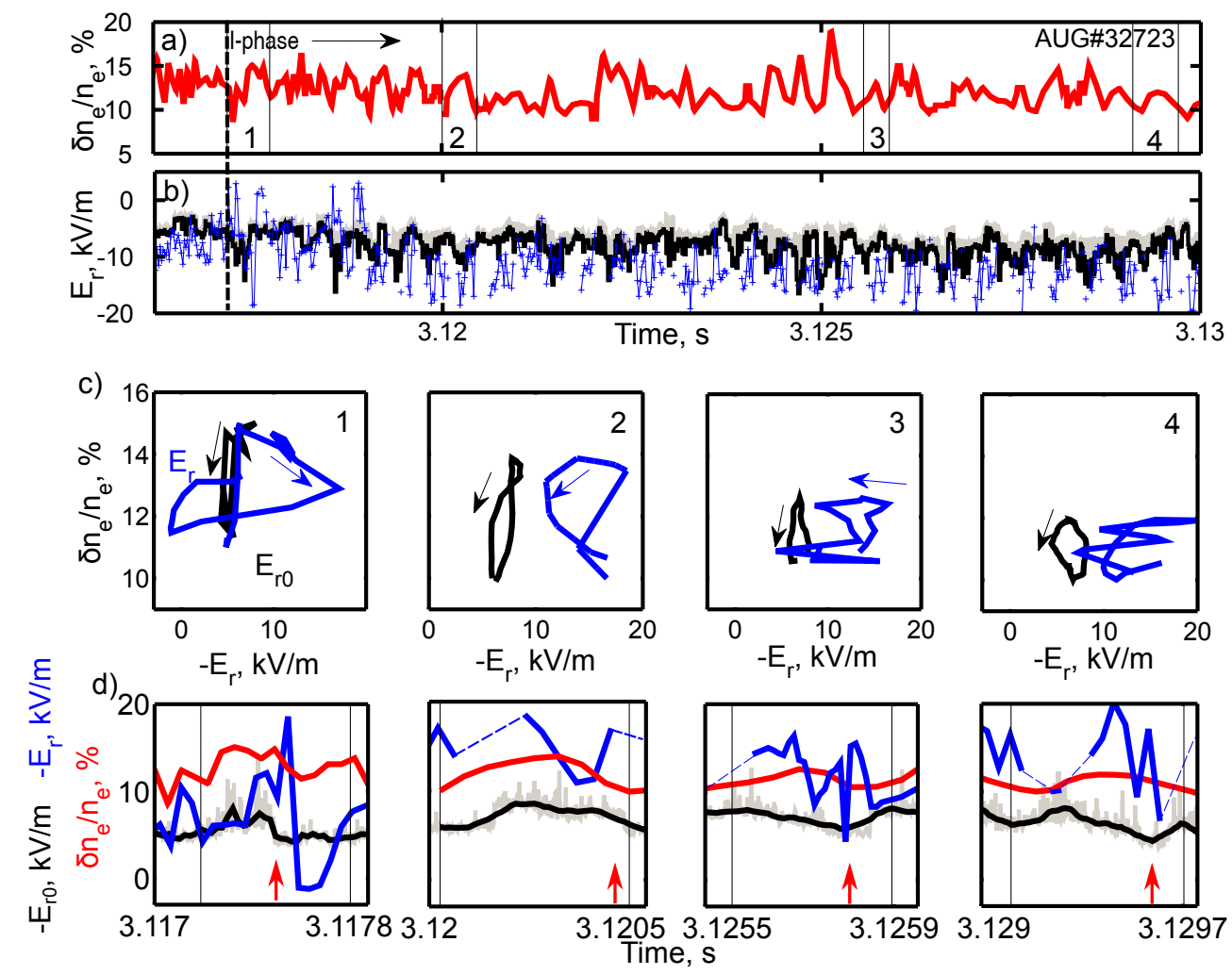

Figure 11. The beginning of the I-phase in \#32723 from the UFSR: a) turbulence level at $\rho_{p o l}=0.98, \mathrm{~b}$ ) the minimum of $E_{r 0}$ (black solid line) and of $E_{r}$ (blue crosses) from Doppler reflectometer, c) the evolution of turbulence level and $E_{r 0}$ (black) or $E_{r}$ (blue dash) amplitudes on the phase plane for individual periods of I-phase and the corresponding time traces d)

small radial movement of plasma.

In between the bursts the estimated $E_{r}$ minimum values are close to $E_{r 0}$ at $\rho_{p o l}=0.97-0.99$. At this stage the absence of a strong phase velocity and of $E_{r}^{Z F}$ can be supposed as both $E_{r}$ and the normalised electron pressure gradient agree and oscillate together with the turbulence level. In this case the I-phase could be explained by edge pressure driven instabilities that lead to a crash of the pedestal pressure with particles expulsion. The edge coherent modes observed around $100 \mathrm{kHz}$ might be precursors and support the relation of the crashes with type-III ELMs [10].

The $E_{r}$ minimum value at the end of the fluctuation bursts shows positive values (Fig. 10b). This fact could be related to the expulsion of filaments accross the separatrix which then are advected in the opposite direction in the SOL. Also the radial velocity of such filaments could lead to positive Doppler shifts. In addition ELMs simulations have shown an inversion of the filaments rotation direction during the crash even inside the separatrix [35], which is also observed in some experiments [36]. Such effects should be studied by full-wave codes in the future.

The beginning of the I-phase needs to be analysed more carefully as it contains 


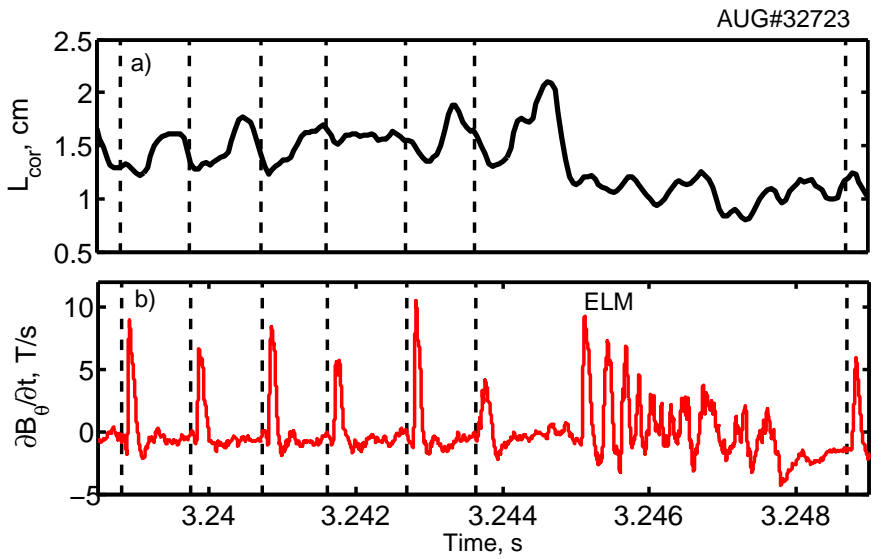

Figure 12. a) Radial correlation length in the pedestal region during the I-phase grows prior to the bursts of $\partial B_{\theta} / \partial t \mathrm{~b}$ ) from the Mirnov coil close to the X-point

irregular oscillations with different phase shift. Figure 11 shows the comparison of the turbulence level, total $E_{r}$ minimum and $E_{r 0}$ in the He plasma discharge \#32723. To analyse the relative phase shift, $E_{r 0}=\nabla p_{e} / 2 e n_{e}$ (Fig. 11b, black solid line) and the density fluctuation level (Fig. 11a) have been taken at the minimum $E_{r 0}$ position around $\rho_{p o l}=0.98$ and the DR data (Fig. $11 \mathrm{p}$, blue crosses) have been treated as mentioned above with windows of $20 \mu \mathrm{s}$. It has been found that for the very first I-phase periods in high (\#32723, Fig. 11) and medium (\#32944) density discharges the turbulence level and $E_{r}$ amplitudes create open loops in the clockwise direction on the phase space plane (Fig. 11k, 1), which are typical for the predator-prey interplay between the flow and the turbulence. In the following periods of the I-phase both $E_{r}$ and $E_{r 0}$ create loops in the counter-clockwise direction showing both the same behavior albeit with wider loops (Fig. 11k, 2-3) for $E_{r}$. After several periods, $E_{r 0}$ and $E_{r}$ synchronise their phases with the turbulence level (Fig. 11k, 4) and the cycles collapse into lines within the given resolution. The time traces (Fig. 11d) show that the bursts of the fluctuation level provoke the relaxation of the $E_{r}$ well and the following increase of the pressure and its gradient first supresses the turbulence and later drives edge instabilities causing the next burst. Note that the $E_{r 0}$ oscillations have a stronger amplitude prior to the bursts and a smaller variation immediatly after the pressure gradient relaxation. The Doppler measurements are only ploted when the signal amplitude allows to extract $E_{r}$ values, they are connected by a dashed line (Fig. 11d).

By constructing the radial correlation length during the I-phase for the UFSR signal amplitude with a time windows of $200 \mu \mathrm{s}$ it has been observed that in-between the bursts the correlation length grows (Fig. $12 \mathrm{a}$ ) at the radial position $\rho_{\text {pol }}=0.98$. The correlation length is estimated as the width of a Gaussian fit of the autocorrelation function. This could indicate the corresponding onset of the coherent modes in the pedestal region. In Fig. 12 the first type I ELM is visible on the magnetic signal at $t=3.245 \mathrm{~s}$ as 


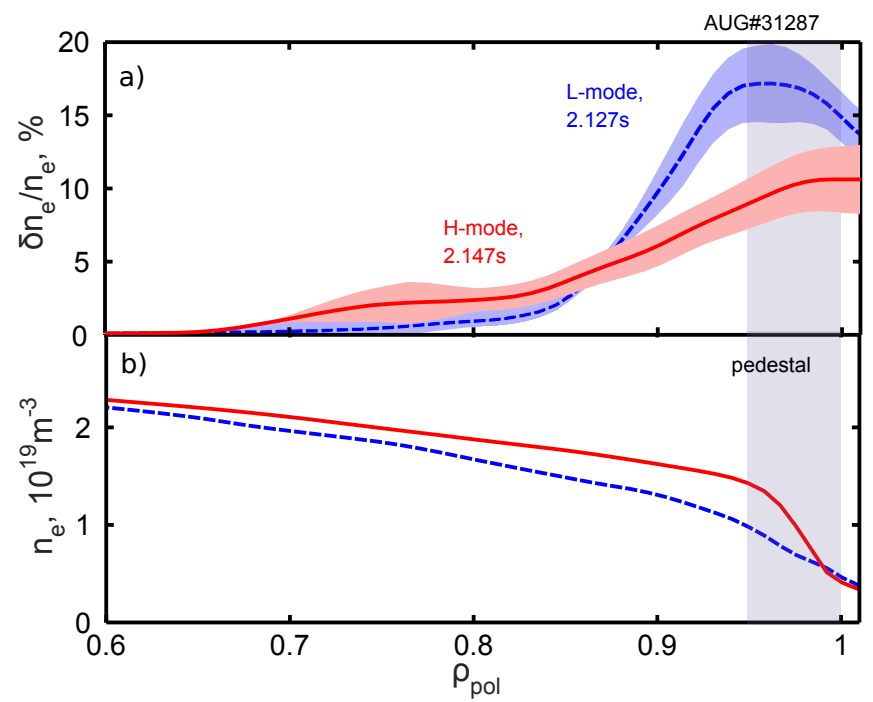

Figure 13. Density fluctuation radial profile a) and density profile b) in L-mode (blue dashed) and in early H-mode (red solid)

a large perturbation. The correlation length reaches $2 \mathrm{~cm}$ prior to the ELM crash. The behaviour is similar before each crash of the I-phase, where the correlation length increases from $1.2 \mathrm{~cm}$ to $1.7 \mathrm{~cm}$.

\section{3. $L-H$ comparison}

In this section an early H-mode (16 ms after the divertor current drop) following the I-phase is compared to an L-mode plasma. To describe the turbulent transport, the radial profiles of the turbulence level have been extracted from the UFSR data. The evolution of the electron density profile is shown in Fig. 2 density profiles build up inside $\rho_{\text {pol }}=0.99$ from shallow in the L-mode $(2.126-2.132 \mathrm{~s})$ to steep in the H-mode (after $2.142 \mathrm{~s}$ ). The profiles presented in Fig. 2 are averaged over $1 \mathrm{~ms}$, which introduces a shallower shape in the pedestal region. Applying a closed-loop method and 1D-wave propagation simulation, the radial wavenumber spectra for each radial position have been found. Then, by an integration over small radial wavenumbers $1 \mathrm{~cm}^{-1}<k_{f}<10$ $\mathrm{cm}^{-1}$ the absolute turbulence level profiles have been reconstructed (Fig. 13). The wavenumber spectra were calculated for radial windows of $3 \mathrm{~cm}$ each, which leads to the smoothing of the turbulence level radial profile. The flattening of the wavenumber spectrum in the L-mode is observed because of the change of turbulence regime as well as because of the saturation effect for the reflectometer phase response in the case of high amplitude density fluctuation [37]. Note that the definition of the spectral slopes might not be accurate due to the non-linear effects and to the spectra saturation in $\mathrm{X}$-mode for high level turbulence [20, 38]. The nonlinearity parameter [20] is estimated as $\gamma=100-500$, although the standard deviation of the phase fluctuation is less than 1 . This indicates that the turbulence measurements were obtained in nonlinear 
regime. Further studies are ongoing to evaluate the sensitivity of the determination of density fluctuation profile to the shape of the input spectra and nonlinear effects. Nevertheless, the absolute turbulence level obtained after integration is not affected by the saturation of the wavenumber spectra [25]. The decrease in turbulence level during the L-H transition confirms the predicted suppression of large scale turbulent structures $\left(0.1<k_{r} \rho_{s}<1\right)$ in the pedestal region, from the separatrix, $\rho_{p o l}=1$, to the top of the density pedestal.

\section{Discussion and conclusions}

First studies of the electron density profile dynamics, turbulence level, $k_{r}$ - and frequency spectra during L-H transitions in ASDEX Upgrade have been performed with a time resolution of $1 \mu \mathrm{s}$. The reconstruction of the turbulence level with a closed-loop procedure needs to be constrained in the spectral indices as it is described in the Section 2. Although the X-mode reflectometry for these discharges might operate in the nonlinear regime, the UFSR measurements indicate that the low $k_{r}$ density fluctuation level decreases in the pedestal region after an L-H transition.

In the observed I-phases an important contribution of the density gradient to the normalised electron pressure gradient $E_{r 0}$ has been confirmed. The I-phase bursts might be explained by growing pressure driven edge instabilities which cause a pressure crash consistent with previous studies on AUG [10]. The edge coherent modes observed in the frequency spectra support this description and can be considered as a precursor, similar to those of type-III ELMs.

The fluctuation level extracted from the UFSR data contains not only the turbulence itself but also the radial density profile movement and the density in expulsed plasma filaments. During the I-phase, the density profile modification due to the burst starts from the pedestal region leading to a simultaneous destruction of the pressure gradient within $0.97<\rho_{\text {pol }}<1$. The crash of the normalised electron pressure gradient $E_{r 0}$, which is taken as a rough estimate of the neoclassical radial electric field, is in phase with turbulence level, pick-up coil bursts and Doppler measured $E_{r}$, which indicates the absence of predator-prey oscillations in the established I-phase. However, electron and ion temperatures can differ in the edge region at the given plasma parameters. Therefore, a complete study of the normalised ion pressure gradient compared to the turbulence level and radial electric field is needed in order to confirm this result, although the achievement of a microsecond time resolution in ion temperature and density measurements is challenging for existing diagnostics.

Some evidences are found that the first few periods of the I-phase could have a different phase behaviour. In the first cycle the normalised electron pressure gradient $E_{r 0}$ deepens together with the drop of the turbulence level, while the Doppler measured $E_{r}$ has a positive phase shift with respect to the turbulence level. First the turbulence starts to grow with the increasing presure gradient, then the strong flow velocity appears and the turbulence level decreases. This could be a consequence of an interplay between 
zonal flow, the background flow and the turbulence, which might have an impact on the transition from L-mode to the I-phase. Note that the phase shift, if there is any, is shorter than 150-200 $\mu \mathrm{s}$, therefore it can be measured only with the diagnostics of a higher temporal resolution. At this point it is important to stress, that this observation is based on Doppler signals which only for short time intervals are strong enough to extract a flow velocity. Further studies are needed to identify the physics of these interesting events and to relate these observations unambiguously to the mean background flow. Nevertheless, the established I-phase in the medium to high densities presents clear features of the ELM-like behaviour.

\section{Acknowledgments}

This work has been carried out within the framework of the Erasmus Mundus International Doctoral College in Fusion Science and Engineering. It has received funding from the Euratom research and training programme 2014-2018 under grant agreement No 633053. The views and opinions expressed herein do not necessarily reflect those of the European Commission. This work was also performed within the framework of the Helmholtz Virtual Institute on Plasma Dynamical Processes and Turbulence Studies using Advanced Microwave Diagnostics.

[1] Wagner F, Becker G, Behringer K, Campbell D, Eberhagen A, Engelhardt W, Fussmann G, Gehre O, Gernhardt J, Gierke G, Haas G, Huang M, Karger F, Keilhacker M, Klüber O, Kornherr M, Lackner K, Lisitano G, Lister G, Mayer H, Meisel D, Müller E, Murmann H, Niedermeyer H, Poschenrieder W, Rapp H, Röhr H, Schneider F, Siller G, Speth E, Stäbler A, Steuer K, Venus G, Vollmer O and Yü Z 1982 Phys. Rev. Lett. 49 1408-1412

[2] Biglari H, Diamond P H and Terry P W 1990 Physics of Fluids B: Plasma Physics 21

[3] Conway G D, Angioni C, Ryter F, Sauter P and Vicente J 2011 Phys. Rev. Lett. 106065001

[4] Zweben S J, Maqueda R J, Hager R, Hallatschek K, Kaye S M, Munsat T, Poli F M, Roquemore A L, Sechrest Y and Stotler D P 2010 Phys. Plasmas 17 -

[5] Estrada T, Ascasíbar E, Happel T, Hidalgo C, Blanco E, Jiménez-Goméz R, Liniers M, LópezBruna D, Tabarés F and Tafalla D 2010 Contributions to Plasma Physics 50 501-506

[6] Xu G S, Wan B N, Wang H Q, Guo H Y, Zhao H L, Liu A D, Naulin V, Diamond P H, Tynan G R, Xu M, Chen R, Jiang M, Liu P, Yan N, Zhang W, Wang L, Liu S C and Ding S Y 2011 Phys. Rev. Lett. 107125001

[7] Schmitz L, Zeng L, Rhodes T L, Hillesheim J C, Doyle E J, Groebner R J, Peebles W a, Burrell K H and Wang G 2012 Phys. Rev. Lett. 108155002

[8] Kobayashi T, Itoh K, Ido T, Kamiya K, Itoh S I, Miura Y, Nagashima Y, Fujisawa a, Inagaki S, Ida K and Hoshino K 2013 Phys. Rev. Lett. 111035002

[9] Cheng J, Dong J Q, Itoh K, Yan L W, Xu M, Zhao K J, Hong W Y, Huang Z H, Ji X Q, Zhong W L, Yu D L, Itoh S I, Nie L, Kong D F, Lan T, Liu a D, Zou X L, Yang Q W, Ding X T, Duan X R and Liu Y 2013 Phys. Rev. Lett. 110265002

[10] Birkenmeier G, Cavedon M, Conway G, Manz P, Stroth U, Fischer R, Fuchert G, Happel T, Laggner F, Maraschek M et al. 2016 Nucl. Fusion 56086009

[11] Shaing K C and Crume E C 1989 Phys. Rev. Lett. 63 2369-2372

[12] Hinton F L and Hazeltine R D 1976 Rev. Mod. Phys. 48 239-308 
[13] Viezzer E, Pütterich T, Angioni C, Bergmann A, Dux R, Fable E, McDermott R, Stroth U, Wolfrum E and the ASDEX Upgrade Team 2014 Nucl. Fusion 54012003

[14] Stroth U, Manz P and Ramisch M 2011 Plas. Phys. and Cont. Fusion 53024006

[15] Itoh S I, Itoh K, Fukuyama A and Miura Y 1991 Phys. Rev. Lett. 672485

[16] Kim E j and Diamond P H 2003 Phys. Rev. Lett. 90185006

[17] Cavedon M, Putterich T, Viezzer E, Birkenmeier G, Happel T, Laggner F M, Manz P, Ryter F, Stroth U and Team T A U 2017 Nucl. Fusion 57014002

[18] Clairet F, Heuraux S, Bottereau C, Molina D, Ducobu L, Leroux F and Barbuti A 2010 Rev. Sci. Instrum. 81 10D903

[19] Varela P, Manso M, Silva A, Team C et al. 2006 Nucl. Fusion 46 S693

[20] Happel T, Görler T, Hennequin P, Lechte C, Bernert M, Conway G, Freethy S, Honoré C, Pinzón J, Stroth U et al. 2017 Plasma Phys. Contr. Fusion 59054009

[21] Willensdorfer M et al. 2014 Plasma Phys. Contr. Fusion 56025008

[22] Bottollier-Curtet H and Ichtchenko G 1987 Rev. Sci. Instrum. 58 539-546

[23] Heuraux S, Hacquin S, da Silva F, Clairet F, Sabot R and Leclert G 2003 Rev. Sci. Instrum. 74 $1501-1505$

[24] Fanack C, Boucher I, Clairet F, Heuraux S, Leclert G and Zou X L 1996 Plasma Phys. Contr. Fusion 381915

[25] Vermare L, Heuraux S, Clairet F, Leclert G and Da Silva F 2006 Nucl. Fusion 46 S743

[26] Gerbaud T 2008 Etude de la microturbulence par réflectométrie dans un plasma de fusion sur le tokamak Tore-Supra Ph.D. thesis Université Henri Poincaré

[27] Zadvitskiy G, Heuraux S, Lechte C, Hacquin S, Sabot R, Clairet F, Gusakov E and Popov A Y 2016 On the possibility to use a fast $2 \mathrm{~d}$ interpretative model for analysis of fusion plasma turbulence from reflectometry data 43rd EPS Conference on Plasma Physics (European Physical Society)

[28] Poli E, Peeters A and Pereverzev G 2001 Comp. Phys. Communications 136 90-104

[29] Viezzer E, Pütterich T, Conway G, Dux R, Happel T, Fuchs J, McDermott R, Ryter F, Sieglin B, Suttrop W, Willensdorfer M, Wolfrum E and the ASDEX Upgrade Team 2013 Nucl. Fusion 53 053005

[30] Vermare L, Hennequin P, Gürcan Ö et al. 2012 Nucl. Fusion 52063008

[31] Ryter F, Pütterich T, Reich M, Scarabosio A, Wolfrum E, Fischer R, Adamov M G, Hicks N, Kurzan B, Maggi C, Neu R, Rohde V, Tardini G and the ASDEX Upgrade TEAM 2009 Nucl. Fusion 49062003

[32] ITER 1999 Nucl. Fusion 39 2175-2249

[33] Silva A, Cupido L, Manso M, Serra F, Nunes I, Santos J, Varela P, Vergamota S, Meneses L, Grossman V et al. 1999 Rev. Sci. Instrum. 70 1072-1075

[34] Yan Z, McKee G, Groebner R, Snyder P, Osborne T and Burrell K 2011 Phys. Rev. Lett. 107 055004

[35] Morales J, Bécoulet M, Garbet X, Orain F, Dif-Pradalier G, Hoelzl M, Pamela S, Huijsmans G, Cahyna P, Fil A et al. 2016 Phys. Plasmas 23042513

[36] Yun G, Lee W, Choi M, Lee J, Park H, Tobias B, Domier C, Luhmann Jr N, Donné A, Lee J et al. 2011 Phys. Rev. Lett. 107045004

[37] Gusakov E, Irzak M, Popov A Y and Teplova N 2015 Plasma Phys. Contr. Fusion 57075009

[38] Gusakov E and Popov A Y 2004 Plasma Phys. Contr. Fusion 461393 